\title{
Calculation of the rise transient and relaxation time of the induced dipole Kerr effect
}

\author{
J. L. Déjardin and P. Blaise \\ Centre d'Etudes Fondamentales, Université de Perpignan, 52 Avenue de Villeneuve, 66860 Perpignan Cedex, France \\ W. T. Coffey \\ Department of Electronic and Electrical Engineering, Trinity College, Dublin 2, Ireland
}

(Received 3 January 1996)

\begin{abstract}
The exact calculation of the rise transient of the birefringence and the corresponding relaxation times by different theoretical methods is described, in particular the Kerr-effect response of an assembly of nonpolar but anisotropically polarizable molecules following the imposition of a constant electric field is studied by solving the Smoluchowski equation. This equation is transformed into a set of differential recurrence relations containing Legendre polynomials of even order only. By taking the Laplace transform of the birefringence function, it is shown that the singularity at $s=0$ (zero-frequency limit) may be removed so that the relaxation time for the rise process may be exactly expressed as a sum of products of Kummer functions and its first derivatives. The second approach is based on a matrix method where the spectrum of eigenvalues $\lambda_{2 j}$ and their associated amplitudes $A_{2 j}$ (extracted from the first components of eigenvectors) are calculated allowing one to express the relaxation time as $\Sigma A_{2 j}\left(\lambda_{2 j}^{-1}\right)$. Numerical values of this time are tabulated for a large range of $g$ values $(0<g<40), g$ being the parameter measuring the ratio of the orientational energy arising from the electrical polarizabilities to the thermal energy. It is thus demonstrated that the lowest eigenvalue $\left(\lambda_{2}\right)$ dominates almost completely the rise process. The effective relaxation time is also calculated exactly and expressed very simply as the ratio of two Kummer functions. Its evolution as a function of $g$ leads to behavior similar to that of the relaxation time obtained either from the Kummer functions or from the eigenvalue method. It is characterized by a maximum situated around $g=2$, which is interesting in view of experimental applications. [S1063-651X(96)01307-4]
\end{abstract}

PACS number(s): 42.70.Df, 05.40.+j, 78.20.Fm, 78.20.Jq

\section{INTRODUCTION}

The exact solution for the dielectric after-effect response following the sudden removal of a dc field for a system of noninteracting polar molecules with uniaxial anisotropy (nematic liquid crystals) has been given by Coffey et al. [1]. This has been accomplished by adapting methods used for the solution of the analogous problem of superparamagnetic relaxation [2]. The calculation proceeds by expanding the solution of the Fokker-Planck equation as a series of Legendre polynomials which in turn leads to differential recurrence relations which govern the decay of the electric polarization or magnetization. This set of differential recurrence relations may be solved exactly in terms of a continued fraction whence the frequency dependence of the susceptibility may be calculated by means of linear response theory. The area under the curve of the decay of the polarization is, since the response is linear, the correlation time $T$ of the first Legendre polynomial. This time $T$ provides a global measure of the relaxation behavior of the system [3]. It has also been demonstrated that $T$ may be expressed in closed form as a sum of products of confluent hypergeometric functions (Kummer functions). In turn $T$ may be expressed exactly in integral form since the product of two Kummer functions may be expressed as an integral [2].

The analysis that we have just given pertains to linear response only since it is confined to the behavior of the first Legendre polynomial in the absence of an applied field. It is the purpose of this paper to show how the method we have developed may also be applied to a nonlinear problem of electro-optics, i.e., the exact calculation of the transient birefringence due to the induced dipole Kerr effect. Thus we shall consider an assembly of nonelectrically interacting nonpolar molecules and we shall suppose that a strong step electric field is applied at the instant $t=0$, and subsequently the molecules only interact through the induced dipole moment. We shall demonstrate how the rise transient of the birefringence may be calculated exactly by expressing the Laplace transform of the birefringence, i.e., the rise transient in terms of continued fractions and we shall demonstrate how the relaxation time defined as the area under the curve of the rise transient may again be exactly expressed in terms of Kummer functions. We remark that this problem, unlike the one considered in Refs. [1,2], is truly nonlinear and so the concept of relaxation time should be used rather than the correlation time. Furthermore, there is no longer any connection between the transient response and the ac response. We also remark due to the symmetry of the potential arising from the induced moments that the differential recurrence relations decouple into two sets, one for the even Legendre polynomials and the other for the odd Legendre polynomials. The analysis described in Refs. [1,2] is for the odd set as the even one is in thermodynamic equilibrium. The opposite situation obtains here where the time behavior of the even set is the quantity of interest. The present problem has also been considered by Watanabe and Morita [4]. However, they did not give exact expressions for the relaxation time, merely calculating that quantity under certain limiting approximations; neither did they solve the three-term recurrence relations for the rise transient in continued fraction form. 
Since the calculation of the relaxation time is not as straightforward as in the dielectric response, we shall first illustrate our method by considering the simple problem of the calculation of the relaxation time of a series inductance $(L)$-resistance $(R)$ circuit when a step field is suddenly applied. Then, we shall transpose this definition to our problem of Kerr-effect relaxation and we shall show how the transient response may be obtained by two methods, one by expressing the Laplace transform of the rise transient as a sum of products of continued fractions and calculating the relaxation time in terms of the zero-frequency limit of this quantity, and the other by expressing the set of differential recurrence relations in the form of a set $\dot{\mathbf{X}}(t)=\mathbf{A X}(t)$, and calculating the eigenvalues and corresponding eigenvectors of this set as detailed in Refs. $[1,2]$. This will verify the exact solutions which we have obtained.

\section{GENERAL EXPRESSIONS FOR THE RISE TIME}

The Fokker-Planck (or Smoluchowski) equation for an assembly of noninteracting and anisotropically polarizable molecules at time $t$ after the imposition of a step electric field $E(t)=E_{0} U(t)(t>0)$ is

$$
2 \tau_{D} \frac{\partial W}{\partial t}=\frac{1}{\sin \vartheta} \frac{\partial}{\partial \vartheta}\left[\sin \vartheta\left(\frac{\partial W}{\partial \vartheta}+\frac{1}{k T} \frac{\partial V}{\partial \vartheta} W\right)\right],
$$

where the orientational potential energy $V(\vartheta)$ is

$$
V(\vartheta)=-k T g U(t) \cos ^{2} \vartheta
$$

$\tau_{D}$ is the Debye dielectric relaxation time, $U(t)$ is the unit step (Heaviside) function, and the nondimensional factor $g$ comparing the potential energy to the thermal energy is defined as

$$
g=\frac{\alpha_{\|}-\alpha_{\perp}}{2 k T} E_{0}^{2}=\frac{\Delta \alpha}{2 k T} E_{0}^{2},
$$

$\alpha_{\|}$and $\alpha_{\perp}$ representing the principal electric polarizabilities parallel and perpendicular to the symmetry axis of the molecule, respectively.

The distribution function $W(\vartheta, t)$ of orientations is axially symmetric and may therefore be expanded as a series of Legendre polynomials to give just as in Refs. $[1,2]$ the differential recurrence relation

$$
\begin{aligned}
& \frac{2 \tau_{D}}{l(l+1)} \dot{f}_{l}+\left[1-\frac{2 g U(t)}{(2 l-1)(2 l+3)}\right] f_{l} \\
& \quad=\frac{2 g(l-1) U(t)}{(2 l-1)(2 l+1)} f_{l-2}-\frac{2 g(l+2) U(t)}{(2 l+1)(2 l+3)} f_{l+2},
\end{aligned}
$$

where

$$
f_{l}(t)=\frac{1}{2 l+1} \frac{a_{l}(t)}{a_{0}}=\left\langle P_{l}(\cos \vartheta)\right\rangle
$$

the angular brackets denoting the expectation value of $P_{l}$ with respect to the distribution $W$, and the $a_{l}$ 's are the Fourier-Laplace coefficients arising from the expansion in zonal harmonics

$$
W(\vartheta, t)=\sum_{l=0}^{\infty} a_{l}(t) P_{l}(\cos \vartheta)
$$

This recurrence relation decouples into odd and even sets, and the even set appropriate to Kerr-effect relaxation is

$$
\begin{aligned}
& \frac{\tau_{D}}{k(2 k+1)} \dot{f}_{2 k}+\left[1-\frac{2 g U(t)}{(4 k-1)(4 k+3)}\right] f_{2 k} \\
& =\frac{2 g(2 k-1) U(t)}{(4 k-1)(4 k+1)} f_{2 k-2}-\frac{2 g(2 k+2) U(t)}{(4 k+1)(4 k+3)} f_{2 k+2} .
\end{aligned}
$$

The Laplace transform of the homogeneous solution of this is

$S_{2 k}(s)$

$$
=\frac{\frac{2 g(2 k-1)}{16 k^{2}-1}}{\frac{s \tau_{D}}{k(2 k+1)}+1-\frac{2 g}{4 k+3}\left(\frac{1}{4 k-1}-\frac{2 k+2}{4 k+1} S_{2 k+2}(s)\right)} \text {, }
$$

where

$$
S_{2 k}(s)=\frac{F_{2 k}(s)}{F_{2 k-2}(s)},
$$

and $F_{2 k}(s)$ is the Laplace transform of $f_{2 k}(t)$ such that

$$
F_{2 k}(s)=\int_{0}^{\infty} f_{2 k}(t) e^{-s t} d t
$$

The inhomogeneous solution is determined by noting that all initial conditions vanish save $k=0$ when $f_{0}(t)=f_{0}(0)=1$, whence we easily find that the Laplace transform of the inhomogeneous solution appropriate for Kerr-effect relaxation $(k=1)$ is

$$
F_{2}(s)=\frac{1}{s} \frac{\frac{2 g}{15}}{\frac{s \tau_{D}}{3}+1-\frac{2 g}{21}+\frac{8 g}{35} S_{4}(s)} .
$$

This is the Laplace transform of the growth of the birefringence. We also note by the final value theorem of Laplace transformation that

$$
\lim _{s \rightarrow 0} F_{2}(s)=\frac{\frac{2 g}{15}}{1-\frac{2 g}{21}+\frac{8 g}{35} S_{4}(0)}=\lim _{t \rightarrow \infty} f_{2}(t)=f_{2}(\infty)
$$

It now remains to extract a formula for the relaxation time from this solution. First, we remark that the Laplace transform (denoted by $\mathcal{L}$ ) of the rise transient is 


$$
\mathcal{L}\left[f_{2}(\infty)-f_{2}(t)\right]=\frac{f_{2}(\infty)}{s}-F_{2}(s)
$$

The area under this curve is

$$
\begin{aligned}
\int_{0}^{\infty}\left[f_{2}(\infty)-f_{2}(t)\right] d t & =\lim _{s \rightarrow 0} \int_{0}^{\infty}\left[f_{2}(\infty)-f_{2}(t)\right] e^{-s t} d t \\
& =\lim _{s \rightarrow 0}\left[\frac{f_{2}(\infty)}{s}-F_{2}(s)\right] .
\end{aligned}
$$

On inspection, this equation would appear to have a singularity at $s=0$. This is not so, however, as we illustrate first of all by considering a series of $R, L$ circuits with an emf $e(t)$ $=E U(t)$ suddenly applied at $t=0$. The instantaneous current $i(t)$ flowing through these passive elements is

$$
i(t)=\frac{e(t)}{R}\left(1-e^{-t / \tau}\right),
$$

whence

$$
\int_{0}^{\infty}[i(\infty)-i(t)] d t=\frac{\tau E}{R}=\tau i(\infty),
$$

and so the time constant $\tau$ characterizing this electrical circuit is

$$
\tau=\frac{1}{i(\infty)} \int_{0}^{\infty}[i(\infty)-i(t)] d t=\frac{1}{i(\infty)} \lim _{s \rightarrow 0}\left[\frac{i(\infty)}{s}-I(s)\right],
$$

where $I(s)$ is the Laplace transform of $i(t)$.

Now,

$$
I(s)=\frac{E}{L} \frac{1}{s(s+1 / \tau)},
$$

so that on decomposition of $I(s)$ into partial fractions we have

$$
\tau=\frac{1}{i(\infty)} \lim _{s \rightarrow 0}\left[\frac{i(\infty)}{s}-i(\infty)\left(\frac{1}{s}-\frac{1}{s+1 / \tau}\right)\right]=\frac{L}{R},
$$

as is well known.
We may use an analogous result for the definition of the relaxation time in our Kerr-effect relaxation problem. We have

$$
\begin{aligned}
T & =\frac{1}{f_{2}(\infty)} \lim _{s \rightarrow 0} \int_{0}^{\infty}\left[f_{2}(\infty)-f_{2}(t)\right] e^{-s t} d t \\
& =\frac{1}{f_{2}(\infty)} \lim _{s \rightarrow 0}\left[\frac{f_{2}(\infty)}{s}-F_{2}(s)\right]=\lim _{s \rightarrow 0}\left[\frac{1}{s}-\frac{F_{2}(s)}{f_{2}(\infty)}\right] .
\end{aligned}
$$

Now,

$$
\frac{F_{2}(s)}{f_{2}(\infty)}=\frac{\frac{2 g}{15} \frac{1}{s}}{s \frac{\tau_{D}}{3}+1-\frac{2 g}{21}+\frac{8 g}{35} S_{4}(s)} \frac{1-\frac{2 g}{21}-\frac{8 g}{35} S_{4}(0)}{\frac{2 g}{15}}
$$

Equation (19), which yields $T$, is not defined for $s=0$. In order to overcome this difficulty, we remark that $S_{4}(s)$ is a continued fraction of the form

$$
\frac{A}{B s+C+D S_{6}(s)},
$$

where $A, B, C$, and $D$ are constants independent of $s$. The function $S_{6}(s)$ is again a continued fraction which depends on $S_{8}(s)$ and so on, so that after reduction to the same denominator, the numerator of $s F_{2}(s)$ will always be one degree less than that of the denominator because of the presence of the first degree term $B s$. This can therefore be written in the polynomial form as

$$
\begin{aligned}
\frac{f_{2}(\infty)}{s} & =\frac{1}{s} \frac{2 g / 15}{1-2 g / 21+(8 g / 35) S_{4}(0)}=\frac{1}{s} \frac{b_{0}}{c_{0}}, \\
F_{2}(s) & =\frac{1}{s} \frac{2 g / 15}{s \tau_{D} / 3+1-2 g / 21+(8 g / 35) S_{4}(s)} \\
& =\frac{1}{s} \frac{b_{0}+b_{1} s+\cdots+b_{n} s^{n}}{c_{0}+c_{1} s+\cdots+c_{n+1} s^{n+1}},
\end{aligned}
$$

where the $b_{n}$ 's and the $c_{n+1}$ 's are the polynomial coefficients of the numerator and the denominator, respectively, so that

$$
\begin{aligned}
T & =\frac{c_{0}}{b_{0}} \lim _{s \rightarrow 0}\left[\frac{1}{s}\left(\frac{b_{0}}{c_{0}}-\frac{b_{0}+b_{1} s+\cdots+b_{n} s^{n}}{c_{0}+c_{1} s+\cdots+c_{n+1} s^{n+1}}\right)\right] \\
& =\frac{c_{0}}{b_{0}} \lim _{s \rightarrow 0}\left[\frac{\left(b_{0} c_{1}-b_{1} c_{0}\right)+s\left(b_{0} c_{2}-b_{2} c_{0}\right)+\cdots+s^{n-1}\left(b_{0} c_{n}-b_{n} c_{0}\right)+b_{0} c_{n+1} s^{n}}{c_{0}\left(c_{0}+c_{1} s+\cdots+c_{n+1} s^{n+1}\right)}\right]=\frac{b_{0} c_{1}-b_{1} c_{0}}{b_{0} c_{0}} .
\end{aligned}
$$

This demonstrates how the apparent singularity at $s=0$ disappears, in such a manner that $T$ may be rendered by the formula

$$
T=\frac{1}{f_{2}(\infty)} \lim _{s \rightarrow 0}\left[\frac{f_{2}(\infty)}{s}-F_{2}(s)\right] .
$$

Equation (22) is very simple and gives the exact relaxation time for the birefringence rise transient. In order to express explicitly the coefficients $b_{0}, b_{1}, c_{0}, c_{1}$, we can expand $S_{4}(s)$ as a Laurent series about the point $s=0$, namely, 


$$
S_{4}(s)=S_{4}(0)+\sum_{1}^{\infty} \frac{s^{n}}{n !} S_{4}^{(n)}(0),
$$

where

$$
S_{4}^{(n)}(0)=\frac{d^{n} S_{4}(s)}{d s^{n}} s=0 .
$$

With the aid of Eqs. (10), (11), and (24) and after some calculation, we obtain

$$
\begin{aligned}
& \frac{f_{2}(\infty)}{s}-F_{2}(s)=\frac{2 g}{15} \frac{1}{s}\left[\frac{1}{1-2 g / 21+(8 g / 35) S_{4}(0)}-\frac{1}{s \tau_{D} / 3+1-2 g / 21+(8 g / 35) S_{4}(s)}\right] \\
&=\frac{2 g}{15} \frac{1}{s} \frac{\left\{\frac{s \tau_{D}}{3}+1-\frac{2 g}{21}+\frac{8 g}{35}\left[S_{4}(0)+\sum_{j=1}^{\infty} \frac{s^{j}}{j !} S_{4}^{(j)}(0)\right]\right\}-\left(1-\frac{2 g}{21}+\frac{8 g}{35} S_{4}(0)\right)}{\left(1-\frac{2 g}{21}+\frac{8 g}{35} S_{4}(0)\right)\left\{\frac{s \tau_{D}}{3}+1-\frac{2 g}{21}+\frac{8 g}{35}\left[S_{4}(0)+\sum_{j=1}^{\infty} \frac{s^{j}}{j !} S_{4}^{(j)}(0)\right]\right\}} \\
&=\frac{2 g}{15} \frac{1}{s} \frac{8 \tau_{D}}{3}+\frac{8 g}{35} s S_{4}^{\prime}(0)+\frac{8 g}{35} \sum_{j=2}^{\infty} \frac{s^{j}}{j !} S_{4}^{(j)}(0) \\
&\left(1-\frac{2 g}{21}+\frac{8 g}{35} S_{4}(0)\right)\left\{\frac{s \tau_{D}}{3}+1-\frac{2 g}{21}+\frac{8 g}{35}\left[S_{4}(0)+\sum_{j=1}^{\infty} \frac{s^{j}}{j !} S_{4}^{(j)}(0)\right]\right\}
\end{aligned}
$$

which on taking the zero-frequency limit $(s \rightarrow 0)$ yields the exact formula

$$
T=\frac{\frac{\tau_{D}}{3}+\frac{8 g}{35} S_{4}^{\prime}(0)}{1-\frac{2 g}{21}+\frac{8 g}{35} S_{4}(0)},
$$

where $S_{4}^{\prime}(0)$ is the limit as $s \rightarrow 0$ of the first derivative of $S_{4}(s)$ with respect to $s$. Equation (26) may also be expressed in closed form [see Eq. (C3) of Ref. [2] ], namely,

$$
S_{n}(0)=1-\frac{M\left((n-1) / 2, n+\frac{1}{2}, g\right)}{M\left((n-1) / 2, n-\frac{1}{2}, g\right)},
$$

where the Kummer (or confluent hypergeometric) function $M(a, b, z)$ is given by

$$
\begin{aligned}
M(a, b, z)= & 1+\frac{a}{b} z+\frac{a(a+1)}{b(b+1)} \frac{z^{2}}{2 !}+\frac{a(a+1)(a+2)}{b(b+1)(b+2)} \frac{z^{3}}{3 !} \\
& +\cdots,
\end{aligned}
$$

whence

$$
S_{4}(0)=1-\frac{M\left(\frac{3}{2}, \frac{9}{2}, g\right)}{M\left(\frac{3}{2}, \frac{7}{2}, g\right)} .
$$

In order to obtain an expression which is convenient for the numerical calculation of $T$, we can write

$$
S_{n}(s)=\frac{A_{n}}{s B_{n}+C_{n}+D_{n} S_{n+2}(s)},
$$

where

$$
\begin{gathered}
A_{n}=2 g \frac{n-1}{4 n^{2}-1}, \quad B_{n}=\frac{2 \tau_{D}}{n(n+1)}, \\
C_{n}=1-\frac{2 g}{(2 n-1)(2 n+3)}, \quad D_{n}=2 g \frac{n+2}{(2 n+1)(2 n+3)},
\end{gathered}
$$

so that the derivative of $S_{n}(s)$ with respect to $s$ can be expressed in the form of a recurrence relation, namely,

$$
\begin{aligned}
S_{n}^{\prime}(0) & =-A_{n} \frac{B_{n}+D_{n} S_{n+2}^{\prime}(0)}{\left[C_{n}+D_{n} S_{n+2}(0)\right]^{2}} \\
& =-\frac{1}{A_{n}}\left[S_{n}(0)\right]^{2}\left[B_{n}+D_{n} S_{n+2}^{\prime}(0)\right],
\end{aligned}
$$

which for $n=4$ becomes equal to (see Appendix A)

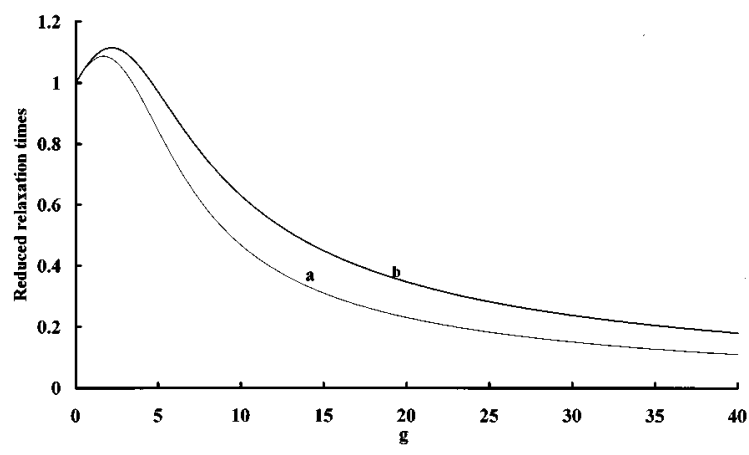

FIG. 1. Comparison of the evolution of the different reduced relaxation times $T^{\prime}$ (curve $a$ ) and $T_{\text {eff }}^{\prime}$ (curve $b$ ) as a function of the electrical anisotropy parameter $g$. Curve $a$ : from Eq. (35), $g_{\max }=1.69$; curve $b$ : from Eq. (49), $g_{\max }=2.18$. 


$$
\begin{aligned}
S_{4}^{\prime}(0)= & -\frac{\tau_{D}}{g}\left[S_{4}(0)\right]^{2}\left\{\frac{21}{20}-\frac{7 \sqrt{\pi}}{32} \sum_{k=1}^{N-1}(-1)^{k+1}(4 k+9) \frac{\Gamma(k+2)}{\Gamma\left(k+\frac{7}{2}\right)} \prod_{j=1}^{k}\left[S_{2 j+4}(0)\right]^{2}\right\} \\
& +(-1)^{N} \frac{7 \sqrt{\pi}}{4} \frac{1}{4 N+7} \frac{\Gamma(N+3)}{\Gamma\left(N+\frac{3}{2}\right)} \prod_{j=1}^{N}\left[S_{2 j+2}(0)\right]^{2} S_{2 N+4}^{\prime}(0)
\end{aligned}
$$

where we have used the gamma function $\Gamma$ defined by

$$
\Gamma(z+1)=z \Gamma(z)
$$

The remainder of Eq. (33) may be set equal to zero for the order $k=N$, so that from a numerical point of view it is sufficient to calculate the alternating series, that is,

$$
S_{4}^{\prime}(0)=-\frac{\tau_{D}}{g}\left[S_{4}(0)\right]^{2}\left\{\frac{21}{20}-\frac{7 \sqrt{\pi}}{32} \sum_{k=1}^{N-1}(-1)^{k+1}(4 k+9) \frac{\Gamma(k+2)}{\Gamma\left(k+\frac{7}{2}\right)} \prod_{j=1}^{k}\left[S_{2 j+4}(0)\right]^{2}\right\} .
$$

We have verified that this equation is rapidly convergent and that a very good precision is obtained for $N=5$. Substituting Eq. (34) into Eq. (26) we can write down the final expression for the reduced relaxation time $T^{\prime}$ normalized to unity,

$$
T^{\prime}=\frac{T}{\tau_{D} / 3}=\frac{1-3\left[S_{4}(0)\right]^{2}\left[\frac{6}{25}-\frac{\sqrt{\pi}}{20} \sum_{k=1}^{N}(-1)^{k+1}(4 k+9) \frac{\Gamma(k+2)}{\Gamma\left(k+\frac{7}{2}\right)} \prod_{j=1}^{k}\left[S_{2 j+4}(0)\right]^{2}\right.}{1-\frac{2 g}{21}+\frac{8 g}{35} S_{4}(0)}
$$

which if so desired may be expressed in terms of Kummer functions as the derivative $S_{2 N+4}^{\prime}(0)$ is no longer involved. The calculation of $T$ ( or $T^{\prime}$ ) is thus much more involved than in the dielectric case. The reverse is true of the Laplace transform of the Kerr function $F_{2}(s)$. The evolution of $T^{\prime}$ is presented in Fig. 1 for different $g$ values varying in the range $0-40$.

\section{CALCULATION OF THE RISE TIME AND RISE TRANSIENT USING MATRIX METHODS}

Having illustrated how one may evaluate the rise transient and the relaxation time by continued fraction methods, we consider how these quantities may be calculated by matrix methods. In order to accomplish this, we arrange our set of recurrence relations in the form

$$
\dot{\mathbf{f}}_{2 k}=-\frac{1}{2 \tau_{D}} \mathbf{M f}_{2 k}+\mathbf{B} U(t), \quad k=1,2, \ldots
$$

where $\dot{\mathbf{f}}_{2 k}, \mathbf{f}_{2 k}$, and $\mathbf{B}$ are column matrices such as

$$
\dot{\mathbf{f}}_{2 k}(t)=\left(\begin{array}{c}
\dot{f}_{2}(t) \\
\dot{f}_{4}(t) \\
\vdots \\
\dot{f}_{2 k}(t) \\
\cdots
\end{array}\right), \quad \mathbf{f}_{2 k}(t)=\left(\begin{array}{c}
f_{2}(t) \\
f_{4}(t) \\
\vdots \\
f_{2 k}(t) \\
\ldots
\end{array}\right), \quad \mathbf{B}=\frac{1}{2 \tau_{D}}\left(\begin{array}{c}
\frac{4 g}{5} \\
0 \\
0 \\
0 \\
\ldots
\end{array}\right)
$$

and $\mathbf{M}$ is an $n \times n$ matrix characteristic of the rise transient birefringence, 


$$
\mathbf{M}=\left(\begin{array}{cccccc}
6\left(1-\frac{2 g}{21}\right) & \frac{48 g}{35} & 0 & 0 & 0 & \ldots \\
-\frac{40 g}{21} & 20\left(1-\frac{2 g}{77}\right) & \frac{80 g}{33} & 0 & 0 & \ldots \\
0 & -\frac{420 g}{143} & 42\left(1-\frac{2 g}{165}\right) & \frac{224 g}{65} & 0 & \ldots \\
0 & 0 & -\frac{336 g}{85} & 72\left(1-\frac{2 g}{285}\right) & 0 & \ldots \\
\ldots & \ldots & \ldots & \ldots & \ldots & \ldots
\end{array}\right) .
$$

The general solution of this matrix equation is

$$
\mathbf{f}_{2 k}(t)=\exp \left(\mathbf{M}^{\prime} t\right) \mathbf{f}_{2 k}(0)+\int_{0}^{t} \exp \left[\mathbf{M}^{\prime}\left(t-t^{\prime}\right) \mathbf{B} U\left(t^{\prime}\right)\right] d t^{\prime},
$$

where

$$
\mathbf{M}^{\prime}=-\frac{1}{2 \tau_{D}} \mathbf{M} .
$$

For the purpose of the calculation of the relaxation time, we are interested in the transient part of this solution which is given by the integral in Eq. (38). For $k=1$, the matrix elements may be expressed in the form

$$
\sum_{j} A_{2 j} \exp \left(-\lambda_{2 j} t\right), \quad j=1,2,3, \ldots
$$

Since we are concerned here with the rise transient, the relaxation modes of the Kerr function may be written as

$$
f_{2}(t)=f_{2}(\infty)\left[1-\sum_{j} A_{2 j} \exp \left(-\lambda_{2 j} t\right)\right],
$$

where the $A_{2 j}$ denote the amplitudes of the first components of the successive eigenvectors associated to the eigenvalues $\lambda_{2 j}$. The longitudinal relaxation time is then the area under this curve, viz.,

$$
\begin{aligned}
T_{\|} & =\lim _{s \rightarrow 0}\left[\frac{1}{s}-\frac{F_{2}(s)}{f_{2}(\infty)}\right]=\lim _{s \rightarrow 0}\left[\frac{1}{s}-\left(\frac{1}{s}-\sum_{k} \frac{A_{2 j}}{s+\lambda_{2 j}}\right)\right] \\
& =\sum_{j} A_{2 j} \lambda_{2 j}^{-1} .
\end{aligned}
$$

In Table I, we present the amplitudes and the eigenvalues of the first four modes for different values of the parameter $g$ up to $g=40$. The eigenvalues arise from the solution of the secular equation

$$
\operatorname{det}\left\{s \mathbf{I}-\mathbf{M}^{\prime}\right\}=0,
$$

where I represents the usual identity matrix.

Since the eigenvalues are presented in the form $\lambda_{2 j} / 6 \tau_{B}$ ( $\tau_{B}$ is the birefringence relaxation time equal to $\tau_{D} / 3$ ), the reduced relaxation time $T_{\|}^{\prime}$ is given by

$$
T_{\|}^{\prime}=\frac{T_{\|}}{\tau_{B}}=6 \sum_{j} A_{2 j} \lambda_{2 j}^{-1} .
$$

We also remark that the amplitudes of rank $j=2 l(l=1,2, \ldots)$ are negative, which was not observed for the dielectric response. Satisfactory convergence was obtained using a $30 \times 30$ matrix in all cases. It is apparent from Table I that the lowest eigenvalue $\lambda_{2}$ provides an accurate enough representation of the relaxation time since the amplitudes of the other modes $\left(A_{4}, A_{6}\right.$, and $\left.A_{8}\right)$ become smaller and smaller in comparison with that of the first one $\left(A_{2}\right)$. This behavior is rather similar to that of the dielectric response governed by $\lambda_{1}, \lambda_{3}$, $\lambda_{5}$, etc. where the mode characterized by $\lambda_{1}$ dominates the response.

\section{COMPARISON OF MATRIX SOLUTIONS WITH THE CONTINUED FRACTION SOLUTIONS}

The solution for the relaxation time rendered by a matrix method which may be implemented with a minimum of analysis agrees exactly (within rounding errors) with the continued fraction solution rendered by Eq. (26) for all $g$ values (see the last column of Table I and the second and fourth columns of Table II). The most interesting result of our theoretical approach is that the solution is again dominated by the first mode $\left(\lambda_{2}\right)$. The Kerr-effect relaxation time, unlike the dielectric one, passes through a maximum at a certain value of $g$ (which may be a useful factor in experiments) before decreasing monotonically to zero for increasing $g$ values. This behavior has also been observed by Morita and Watanabe (see their Fig. 4 in Ref. [4]).

We also compare our solution for the rise transient for the relaxation time with that rendered by the effective eigenvalue. This method may be used here since the rise transient of the electric birefringence for the pure induced dipole mechanism may be approximated by a single exponential term [4] characterized by an effective relaxation time $T_{\text {eff }}$. It is well known, indeed, that when the orientation of the molecules is only due to the anisotropy of the electrical polarizabilities, the Kerr-effect response may be considered as linear-at least for weak fields-according to Kerr's law which relates the birefringence $\Delta n$ to the square electric field $E^{2}$. On the contrary, this is not true for the process arising from pure permanent (field-off) moments which is, in essence, a nonlinear response and needs two effective relaxation times.

Thus the time behavior of the orientational factor may be written as 
TABLE I. Eigenvalues $\lambda_{2 j}$ in the form $\lambda_{2 j} / 6 \tau_{B}$ of the first four modes of the rise of the birefringence as a value of the parameter $g$ and corresponding amplitudes $A_{2 j}, j=1,2,3 \ldots\left[T_{\|}^{\prime}\right.$ is the reduced relaxation time given by Eq. (40b)]. E denotes values of $10^{-n}$.

\begin{tabular}{|c|c|c|c|c|c|c|c|c|c|}
\hline$g$ & $\lambda_{2}$ & $\lambda_{4}$ & $\lambda_{6}$ & $\lambda_{8}$ & $A_{2}$ & $A_{4}$ & $A_{6}$ & $A_{8}$ & $T_{\|}^{\prime}$ \\
\hline 1 & 5.6143 & 19.6181 & 41.6215 & 71.6229 & 1.0037778 & $-3.7818 \mathrm{E}-03$ & $3.9611 \mathrm{E}-06$ & $-1.8553 \mathrm{E}-09$ & 1.0715775 \\
\hline 2 & 5.5955 & 19.5135 & 41.5049 & 71.5026 & 1.0149272 & $-1.4989 \mathrm{E}-02$ & $6.2075 \mathrm{E}-05$ & $-1.1577 \mathrm{E}-07$ & 1.0837014 \\
\hline 3 & 5.9333 & 19.6898 & 41.6521 & 71.6400 & 1.0346603 & $-3.4981 \mathrm{Ee}-02$ & $3.2255 \mathrm{E}-04$ & $-1.3469 \mathrm{E}-06$ & 1.0356783 \\
\hline 4 & 6.6126 & 20.1517 & 42.0657 & 72.0368 & 1.0657605 & $-6.6839 \mathrm{E}-02$ & $1.0866 \mathrm{E}-03$ & $-8.0264 \mathrm{E}-06$ & 0.9472779 \\
\hline 5 & 7.6121 & 20.9061 & 42.7493 & 72.6952 & 1.1117499 & -0.1146148 & $2.8980 \mathrm{E}-03$ & $-3.3285 \mathrm{E}-05$ & 0.8438085 \\
\hline 6 & 8.9031 & 21.9636 & 43.7075 & 73.6180 & 1.1758221 & -0.1823589 & $6.6451 \mathrm{E}-03$ & $-1.0943 \mathrm{E}-04$ & 0.7434993 \\
\hline 7 & 10.4483 & 23.3388 & 44.9461 & 74.8085 & 1.2595970 & -0.2729550 & 0.0136593 & -0.0003051 & 0.6549570 \\
\hline 8 & 12.2020 & 25.0504 & 46.4721 & 76.2709 & 1.3617338 & -0.3867915 & 0.0257966 & -0.0007510 & 0.5802222 \\
\hline 9 & 14.1122 & 27.1189 & 48.2945 & 78.0098 & 1.4768501 & -0.5206982 & 0.0454896 & -0.0016759 & 0.5182252 \\
\hline 10 & 16.1255 & 29.5606 & 50.4239 & 80.0308 & 1.5958169 & -0.6681915 & 0.0757413 & -0.0034537 & 0.4669084 \\
\hline 11 & 18.1945 & 32.3799 & 52.8738 & 82.3401 & 1.7082491 & -0.8217917 & 0.1200035 & -0.0066632 & 0.4241935 \\
\hline 12 & 20.2839 & 35.5630 & 55.6606 & 84.9450 & 1.8061476 & -0.9762882 & 0.1818617 & -0.0121589 & 0.3883119 \\
\hline 13 & 22.3724 & 39.0748 & 58.8040 & 87.8538 & 1.8861301 & -1.1302985 & 0.2644251 & -0.0211482 & 0.3578571 \\
\hline 14 & 24.4500 & 42.8623 & 62.3267 & 91.0759 & 1.9489763 & -1.2848290 & 0.3694000 & -0.0352696 & 0.3317403 \\
\hline 15 & 26.5144 & 46.8604 & 66.2515 & 94.6225 & 1.9976586 & -1.4402463 & 0.4960639 & -0.0566545 & 0.3091245 \\
\hline 16 & 28.5666 & 51.0004 & 70.5967 & 98.5066 & 2.0355681 & -1.5940234 & 0.6407689 & -0.08794 & 0.2893651 \\
\hline 17 & 30.6088 & 55.2187 & 75.3691 & 102.7434 & 2.0656030 & -1.7407382 & 0.7977409 & -0.1322293 & 0.2719609 \\
\hline 18 & 32.6432 & 59.4649 & 80.5565 & 107.3511 & 2.0899352 & -1.8742132 & 0.9611777 & -0.1928122 & 0.2565194 \\
\hline 19 & 34.6717 & 63.7051 & 86.1241 & 112.3509 & 2.1100950 & -1.9901502 & 1.1272926 & -0.2727670 & 0.2427288 \\
\hline 20 & 36.6956 & 67.9216 & & & & & & -0.3742051 & 0.2303396 \\
\hline 21 & 38.7161 & 72.1086 & 98.1590 & 123.6214 & 2.1417853 & -2.1675238 & 1.4626139 & -0.4973936 & 0.2191496 \\
\hline 22 & 40.7338 & 76.2668 & 104.4757 & 129.9351 & 2.1545532 & -2.2333368 & 1.6292428 & -0.6401156 & 0.2089935 \\
\hline 23 & 42.7493 & 80.4001 & 110.8907 & 136.7161 & 2.1658070 & -2.2878857 & 1.7903944 & -0.7978828 & 0.1997347 \\
\hline 24 & 44.7630 & 84.5130 & 117.3409 & 143.9543 & 2.1758180 & -2.3337847 & 1.9406222 & -0.9652822 & 0.1912598 \\
\hline 25 & 46.7752 & 88.6095 & 123.7815 & 151.6162 & 2.1847923 & -2.3730623 & 2.0752071 & -1.1377944 & 0.1834734 \\
\hline 26 & 48.7862 & 92.6929 & 130.1864 & 159.6444 & 2.1928902 & -2.4072134 & 2.1917859 & -1.3127503 & 0.1762952 \\
\hline 27 & 50.7961 & 96.7659 & 136.5449 & 167.9631 & 2.2002386 & -2.4373190 & 2.2906204 & -1.4886926 & 0.1696567 \\
\hline 28 & 52.8052 & 100.8305 & 142.8565 & 176.4865 & 2.2069402 & -2.4641625 & 2.3737646 & -1.6637680 & 0.1634994 \\
\hline 29 & 54.8134 & 104.8881 & 149.1256 & 185.1296 & 2.2130789 & -2.4883201 & 2.4439729 & -1.8345247 & 0.1577728 \\
\hline 30 & 56.8210 & 108.9398 & 155.3586 & 193.8182 & 2.2187244 & -2.5102254 & 2.5039219 & -1.9961035 & 0.1524333 \\
\hline 31 & 58.8279 & 112.9867 & 161.5618 & 202.4964 & 2.2239349 & -2.5302124 & 2.5558515 & -2.1436996 & 0.1474431 \\
\hline 32 & 60.8343 & 117.0294 & 167.7405 & 211.1292 & 2.2287596 & -2.5485448 & 2.6014952 & -2.2741947 & 0.1427689 \\
\hline 33 & 62.8403 & 121.0685 & 173.8992 & 219.6999 & 2.2332405 & -2.5654347 & 2.6421443 & -2.3868793 & 0.1383817 \\
\hline 34 & 64.8458 & 125.1044 & 180.0413 & 228.2050 & 2.2374136 & -2.5810564 & 2.6787484 & -2.4830607 & 0.1342559 \\
\hline 35 & 66.8510 & 129.1376 & 186.1696 & 236.6489 & 2.2413099 & -2.5955549 & 2.7120094 & -2.5651318 & 0.1303689 \\
\hline 36 & 68.8558 & 133.1683 & 192.2862 & 245.0391 & 2.2449563 & -2.6090526 & 2.7424546 & -2.6357225 & 0.1267004 \\
\hline 37 & 70.8603 & 137.1968 & 198.3927 & 253.3839 & 2.2483765 & -2.6216535 & 2.7704885 & -2.6971996 & 0.1232326 \\
\hline 38 & 72.8646 & 141.2234 & 204.4907 & 261.6906 & 2.2515909 & -2.6334472 & 2.7964291 & -2.7514793 & 0.1199495 \\
\hline 39 & 74.8686 & 145.2483 & 210.5811 & 269.9658 & 2.2546174 & -2.6445107 & 2.8205305 & -2.8000363 & 0.1168366 \\
\hline 40 & 76.8723 & 149.2716 & 216.6648 & 278.2145 & 2.2574733 & -2.6549129 & 2.8430046 & -2.8439603 & 0.1138812 \\
\hline
\end{tabular}

$$
f_{2}(t)=\left\langle P_{2}(\cos \vartheta)\right\rangle(t)=f_{2}(\infty)\left[1-\sum_{j} A_{2 j} \exp \left(-\lambda_{2 j} t\right)\right]
$$

which in terms of the effective eigenvalue $\lambda_{\text {eff }}$ becomes

$$
f_{2}(t)=f_{2}(\infty)\left[1-\exp \left(-\lambda_{\text {eff }} t\right)\right],
$$

so that the effective relaxation time $T_{\text {eff }}$ is then

$$
T_{\text {eff }}=\lambda_{\text {eff }}^{-1}=\frac{\left\langle P_{2}(\cos \vartheta)\right\rangle(0)}{\left\langle P_{2}(\cos \vartheta)\right\rangle(\infty)}=\frac{\dot{f}_{2}(0)}{f_{2}(\infty)}=\left(\sum_{j} A_{2 j} \lambda_{2 j}\right)^{-1}
$$

and the reduced effective relaxation time is

$$
T_{\mathrm{eff}}^{\prime}=\frac{T_{\mathrm{eff}}}{\tau_{B}}=6\left(\sum_{j} A_{2 j} \lambda_{2 j}\right)^{-1} .
$$

From Table II, it is apparent that $T_{\text {eff }}^{\prime}$ given by Eq. (43b) based on the effective eigenvalue technique is a little different from the numerical values of the relaxation times obtained using continued fraction or matrix methods, and so does not provide a rigorous representation of the rise transient birefringence phenomenon. In particular, this method leads to higher values of the relaxation time as $g$ becomes greater than 1. As already mentioned by Coffey, Kalmykov, 
TABLE II. Table of values of the reduced relaxation times from the solutions given by Eqs. (35) and (43b).

\begin{tabular}{cccccc}
\hline \hline$g$ & $T^{\prime}$ & $T_{\text {eff }}^{\prime}$ & $g$ & $T^{\prime}$ & $T_{\text {eff }}^{\prime}$ \\
\hline 0.2 & 1.018240 & 1.018509 & 13.0 & 0.357781 & 0.507055 \\
0.4 & 1.034718 & 1.035819 & 14.0 & 0.331624 & 0.475740 \\
0.6 & 1.049221 & 1.051753 & 15.0 & 0.308955 & 0.447948 \\
0.8 & 1.061562 & 1.066146 & 16.0 & 0.289129 & 0.423142 \\
1.0 & 1.071578 & 1.078845 & 17.0 & 0.271646 & 0.400883 \\
1.2 & 1.079141 & 1.089724 & 18.0 & 0.256111 & 0.380807 \\
1.4 & 1.084161 & 1.098675 & 19.0 & 0.242216 & 0.362616 \\
1.6 & 1.086591 & 1.105625 & 20.0 & 0.229710 & 0.346062 \\
1.8 & 1.086425 & 1.110525 & 21.0 & 0.218392 & 0.330936 \\
2.0 & 1.083701 & 1.113363 & 22.0 & 0.208099 & 0.317064 \\
2.2 & 1.078499 & 1.114155 & 23.0 & 0.198696 & 0.304297 \\
2.4 & 1.070933 & 1.112948 & 24.0 & 0.190070 & 0.292511 \\
2.6 & 1.061154 & 1.109817 & 25.0 & 0.182128 & 0.281597 \\
2.8 & 1.049337 & 1.104860 & 26.0 & 0.174790 & 0.271463 \\
3.0 & 1.035678 & 1.098195 & 27.0 & 0.167988 & 0.262029 \\
3.2 & 1.020389 & 1.089958 & 28.0 & 0.161667 & 0.253225 \\
3.4 & 1.003688 & 1.080292 & 29.0 & 0.155775 & 0.244990 \\
3.6 & 0.985793 & 1.069351 & 30.0 & 0.150271 & 0.237272 \\
3.8 & 0.966921 & 1.057290 & 31.0 & 0.145117 & 0.230023 \\
4.0 & 0.947278 & 1.044262 & 32.0 & 0.140281 & 0.223202 \\
5.0 & 0.843808 & 0.969599 & 33.0 & 0.135734 & 0.216772 \\
6.0 & 0.743499 & 0.889454 & 34.0 & 0.131452 & 0.210701 \\
7.0 & 0.654956 & 0.812812 & 35.0 & 0.127411 & 0.204960 \\
8.0 & 0.580220 & 0.743534 & 36.0 & 0.123592 & 0.199523 \\
9.0 & 0.518219 & 0.682521 & 37.0 & 0.119978 & 0.194366 \\
10.0 & 0.466895 & 0.629319 & 38.0 & 0.116553 & 0.189468 \\
11.0 & 0.424168 & 0.582994 & 39.0 & 0.113302 & 0.184810 \\
12.0 & 0.388266 & 0.542545 & 40.0 & 0.110213 & 0.180375 \\
\hline \hline & & & & & \\
& & & & \\
\hline
\end{tabular}

and Massawe [5], this is due to the loss of information on the different time regions of relaxation that are involved. In fact, the second expression in Eq. (43a) represents the initial slope of the rise of the birefringence, which is always interesting experimentally. It is also possible to find another expression for $T_{\text {eff }}$ starting from the set of differential recurrence equations. This has the advantage just as in the transverse dielectric response [1] of providing a simple analytic formula for the relaxation time. Setting $k=1$ in Eq. (6), one obtains

$$
\dot{f}_{2}(t)=-\frac{1}{2 \tau_{D}}\left[6\left(1-\frac{2 g}{21}\right) f_{2}(t)+\frac{48 g}{35} f_{4}(t)\right]+\frac{2 g}{5 \tau_{D}},
$$

which for $t=0$ reduces to

$$
\dot{f}_{2}(0)=\frac{2 g}{5 \tau_{D}}
$$

since $f_{2}(0)=f_{4}(0)=0$ (initial conditions).

It remains to evaluate the asymptotic value attained by $f_{2}(t)$ as $t \rightarrow \infty$, i.e., $f_{2}(\infty)$. The details of that calculation are given in Appendix B. It is found that [6]

$$
f_{2 k}(\infty)=\frac{g^{k}}{2} \frac{\Gamma\left(k+\frac{1}{2}\right)}{\Gamma\left(2 k+\frac{3}{2}\right)} \frac{M\left(k+\frac{1}{2}, 2 k+\frac{3}{2}, g\right)}{M\left(\frac{1}{2}, \frac{3}{2}, g\right)},
$$

so that for $k=1$, one has

$$
f_{2}(\infty)=\frac{2 g}{15} \frac{M\left(\frac{3}{2}, \frac{7}{2}, g\right)}{M\left(\frac{1}{2}, \frac{3}{2}, g\right)}=1-\frac{M\left(\frac{1}{2}, \frac{5}{2}, g\right)}{M\left(\frac{1}{2}, \frac{3}{2}, g\right)}
$$

This equation coincides rigorously with that obtained in another form by Watanabe and Morita in their study of Kerreffect relaxation in high electric fields [4], but the essential lines of the calculation are not given. Their expression is

$$
\lim _{t \rightarrow \infty}\left\langle P_{2}(\cos \vartheta)\right\rangle(t)=\frac{1}{2}\left[\frac{M\left(\frac{3}{2}, \frac{5}{2}, g\right)}{M\left(\frac{1}{2}, \frac{3}{2}, g\right)}-1\right] .
$$

Substituting Eqs. (45) and (47) into the second expression of Eq. (43a), one finally finds that the effective relaxation time may be expressed exactly as the ratio of two Kummer functions, namely,

$$
T_{\mathrm{eff}}^{\prime}=\frac{T_{\mathrm{eff}}}{\tau_{D} / 3}=\frac{M\left(\frac{3}{2}, \frac{7}{2}, g\right)}{M\left(\frac{1}{2}, \frac{3}{2}, g\right)},
$$

where $T_{\text {eff }}^{\prime}$ represents a reduced relaxation time equal to 1 as $g=0$. The variations of this time against $g$ are plotted in Fig. 1. One remarks that the general behavior of this curve resembles that of $T^{\prime}(g)$, again possessing a maximum but slightly shifted on the right. Both these plots start from $T^{\prime}=1$ when $g=0$, and have a maximum situated about $g=2$, and then pass again through a particular value of $g(3<g<5)$ where $T^{\prime}=1$. This may be explained as follows: it is well known that the stationary state birefringence for low fields is simply given by $2 g / 15$, which corresponds to Kerr's law. Equation (47) calculated for any value of the electrical parameter $g$ shows that this stationary state is modulated by the ratio of two Kummer functions, involving deviation from Kerr's law. In particular, this ratio is greater than 1 in the range $0<g<5$. Such information may be exploited experimentally.

\section{APPENDIX A: CALCULATION OF THE LAPLACE TRANSFORM $S_{4}^{\prime}(0)$}

From Eq. (32) we have

$$
\begin{aligned}
S_{n}^{\prime}(0)= & -\left[S_{n}(0)\right]^{2}\left[\frac{\tau_{D}}{g} \frac{4 n^{2}-1}{(n-1) n(n+1)}\right. \\
& \left.+\frac{(n+2)(2 n-1)}{(n-1)(2 n+3)} S_{n+2}^{\prime}(0)\right] .
\end{aligned}
$$

Now, the next function will be

$$
\begin{aligned}
S_{n+2}^{\prime}(0)= & -\left[S_{n+2}(0)\right]^{2}\left[\frac{\tau_{D}}{g} \frac{(2 n+3)(2 n+5)}{(n+1)(n+2)(n+3)}\right. \\
& \left.+\frac{(n+4)(2 n+3)}{(n+1)(2 n+7)} S_{n+4}^{\prime}(0)\right]
\end{aligned}
$$

which by substitution into Eq. (A1) yields 


$$
\begin{aligned}
S_{n}^{\prime}(0)= & -\frac{\tau_{D}}{g}\left[S_{n}(0)\right]^{2}\left\{\frac{(2 n-1)(2 n+1)}{(n-1) n(n+1)}-\frac{(n+2)(2 n-1)(2 n+3)(2 n+5)}{(n-1)(n+1)(n+2)(n+3)(2 n+3)}\left[S_{n+2}(0)\right]^{2}\right\} \\
& +\frac{(n+2)(n+4)(2 n-1)(2 n+3)}{(n-1)(n+1)(2 n+3)(2 n+7)}\left[S_{n}(0)\right]^{2}\left[S_{n+2}(0)\right]^{2} S_{n+4}^{\prime}(0) .
\end{aligned}
$$

Since we are only interested here with even values of $n$, we can set $n=2 m(m=1,2, \ldots)$ so that Eq. (A3) may be written as

$$
\begin{aligned}
S_{2 m}^{\prime}(0)= & -\frac{\tau_{D}}{g} \frac{(4 m-1)(4 m+1)}{(2 m-1) 2 m(2 m+1)}\left[S_{2 m}(0)\right]^{2}+\left[S_{2 m}(0)\right]^{2} \sum_{k=1}^{N-1}(-1)^{k+1} \\
& \times \frac{(4 m+4 k+1) ! !}{(4 m-3) ! !} \frac{(4 m-1) ! !}{(4 m+4 k-1) ! !} \frac{(2 m-3) ! !}{(2 m+2 k+1) ! !} \frac{(2 m+2 k-2) ! !}{(2 m) ! !} \prod_{j=1}^{k}\left[S_{2 j+2 m}(0)\right]^{2} \\
& +(-1)^{N} \frac{4 m-1}{4 m+4 N-1} \frac{(2 m-3) ! !}{(2 m+2 N-3) ! !} \frac{(2 m+2 N) ! !}{(2 m) ! !} \prod_{j=1}^{N}\left[S_{2 j+2}(0)\right]^{2} S_{2 N+2 m}^{\prime}(0)
\end{aligned}
$$

On noting that [7]

$$
\begin{gathered}
\Gamma\left(n+\frac{1}{2}\right)=\frac{(2 n-1) ! !}{2^{n}} \sqrt{\pi}, \\
(2 m+2 n) ! !=2^{m+n}(m+n) !,
\end{gathered}
$$

and setting $m=2$ in Eq. (A4), we find the desired result for $S_{4}^{\prime}(0)$ given by Eq. (33).

\section{APPENDIX B: EVALUATION OF THE ASYMPTOTIC LIMIT $f_{2 n}(\infty)$ AS A RATIO OF TWO KUMMER FUNCTIONS}

For the rise transient process, we can adopt the following definition $(x=\cos \vartheta)$ :

$$
\begin{aligned}
f_{2 n}(\infty) & =\frac{\int_{-1}^{+1} P_{2 n}(x) \exp \left(g x^{2}\right) d x}{\int_{-1}^{+1} \exp \left(g x^{2}\right) d x} \\
& =\frac{1}{2 M\left(\frac{1}{2}, \frac{3}{2}, g\right)} \int_{-1}^{+1} P_{2 n}(x) \exp \left(g x^{2}\right) d x,
\end{aligned}
$$

which coincides with the initial condition of the decay process. Then, we can use the same method as that employed by Coffey et al. [2] for the magnetic susceptibility and adapt it to even Legendre polynomials. We have

$$
\exp \left(g x^{2}\right)=\sum_{n=0}^{\infty} \frac{g^{n}}{n !} x^{2 n}
$$

and

$$
x^{2 n}=\sum_{k=0}^{n}(4 k+1) \frac{(2 n) !}{(2 n+2 k+1) !} 2^{2 k} \frac{(n+k) !}{(n-k) !} P_{2 k}(x)
$$

By using the orthogonality property of the Legendre polynomials and the definition of the Kummer function [Eq. (28)], we obtain $(k=n+N)$

$$
\begin{aligned}
f_{2 n}(\infty) & =\frac{2^{2 n}}{M\left(\frac{1}{2}, \frac{3}{2}, g\right)} \sum_{k=n}^{\infty} \frac{g^{k}}{k !} \frac{(2 k) !(n+k) !}{(2 n+2 k+1) !(k-n) !} \\
& =\frac{g^{n}}{2 M\left(\frac{1}{2}, \frac{3}{2}, g\right)} \sum_{N=0}^{\infty} \frac{g^{N}}{N !} \frac{\Gamma\left(n+N+\frac{1}{2}\right)}{\Gamma\left(2 n+N+\frac{3}{2}\right)} \\
& =\frac{g^{n}}{2 M\left(\frac{1}{2}, \frac{3}{2}, g\right)} \frac{\Gamma\left(n+\frac{1}{2}\right)}{\Gamma\left(2 n+\frac{3}{2}\right)} M\left(n+\frac{1}{2}, 2 n+\frac{3}{2}, g\right),
\end{aligned}
$$

so that for $n=1$

$$
f_{2}(\infty)=\frac{g}{2 M\left(\frac{1}{2}, \frac{3}{2}, g\right)} \frac{\Gamma\left(\frac{3}{2}\right)}{\Gamma\left(\frac{7}{2}\right)} M\left(\frac{3}{2}, \frac{7}{2}, g\right)=\frac{2 g}{15} \frac{M\left(\frac{3}{2}, \frac{7}{2}, g\right)}{M\left(\frac{1}{2}, \frac{3}{2}, g\right)},
$$

that is, Eq. (47).
[1] W. T. Coffey, D. S. F. Crothers, Yu. P. Kalmykov, and J. T. Waldron, Physica A 213, 551 (1995).

[2] W. T. Coffey, D. S. F. Crothers, Yu. P. Kalmykov, E. S. Massawe, and J. T. Waldron, Phys. Rev. E 49, 1869 (1994).

[3] M. San Miguel, L. Pesquera, M. A. Rodrigues, and A. Hernàndez-Machado, Phys. Rev. A 35, 208 (1987).
[4] H. Watanabe and A. Morita, Adv. Chem. Phys. 56, 255 (1984).

[5] W. T. Coffey, Yu. P. Kalmykov, and E. S. Massawe, Adv. Chem. Phys. 85, 667 (1993).

[6] P. M. Déjardin (private communication).

[7] Handbook of Mathematical Functions, edited by $\mathrm{M}$. Abramowitz and I. Stegun (Dover, New York, 1964). 